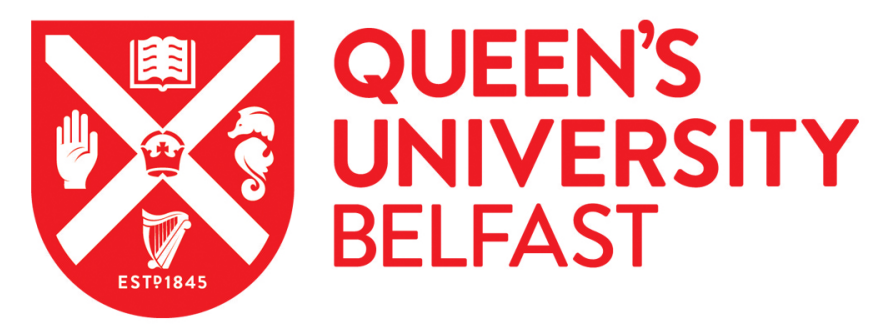

\title{
Strong coupling in molecular exciton-plasmon Au nanorod array systems
}

Pollard, R., Murphy, A., Fedele, S., Hakami, M., \& Rice, J. (2016). Strong coupling in molecular exciton-plasmon Au nanorod array systems. Applied Physics Letters, 108(5). https://doi.org/10.1063/1.4941078

Published in:

Applied Physics Letters

Document Version:

Publisher's PDF, also known as Version of record

Queen's University Belfast - Research Portal:

Link to publication record in Queen's University Belfast Research Portal

Publisher rights

Copyright 2016 AIP. This work is made available online in accordance with the publisher's policies. Please refer to any applicable terms of use of the publisher

\section{General rights}

Copyright for the publications made accessible via the Queen's University Belfast Research Portal is retained by the author(s) and / or other copyright owners and it is a condition of accessing these publications that users recognise and abide by the legal requirements associated with these rights.

Take down policy

The Research Portal is Queen's institutional repository that provides access to Queen's research output. Every effort has been made to ensure that content in the Research Portal does not infringe any person's rights, or applicable UK laws. If you discover content in the Research Portal that you believe breaches copyright or violates any law, please contact openaccess@qub.ac.uk. 


\section{Strong coupling in molecular exciton-plasmon Au nanorod array systems}

Stefano Fedele, Manal Hakami, Antony Murphy, Robert Pollard, and James Rice

Citation: Appl. Phys. Lett. 108, 053102 (2016); doi: 10.1063/1.4941078

View online: https://doi.org/10.1063/1.4941078

View Table of Contents: http://aip.scitation.org/toc/apl/108/5

Published by the American Institute of Physics

\section{Articles you may be interested in}

Plasmon enhanced fluorescence studies from aligned gold nanorod arrays modified with $\mathrm{SiO}_{2}$ spacer layers Applied Physics Letters 106, 183109 (2015); 10.1063/1.4919968

Plasmonic properties of gold nanoparticles on silicon substrates: Understanding Fano-like spectra observed in reflection

Applied Physics Letters 109, 111901 (2016); 10.1063/1.4962731

Strong coupling between Tamm plasmon polariton and two dimensional semiconductor excitons Applied Physics Letters 110, 051101 (2017); 10.1063/1.4974901

Electromagnetic fields around silver nanoparticles and dimers

The Journal of Chemical Physics 120, 357 (2004); 10.1063/1.1629280

Couple molecular excitons to surface plasmon polaritons in an organic-dye-doped nanostructured cavity Applied Physics Letters 108, 193111 (2016); 10.1063/1.4949562

Core-shell gold J-aggregate nanoparticles for highly efficient strong coupling applications Applied Physics Letters 96, 253107 (2010); 10.1063/1.3456523

\section{Conference Proceedings}




\title{
Strong coupling in molecular exciton-plasmon Au nanorod array systems
}

\author{
Stefano Fedele, ${ }^{1}$ Manal Hakami, ${ }^{1}$ Antony Murphy, ${ }^{2}$ Robert Pollard,${ }^{2}$ and James Rice ${ }^{1, a)}$ \\ ${ }^{1}$ School of Physics, University College Dublin, Belfield, Dublin 4, Ireland \\ ${ }^{2}$ Centre for Nanostructured Media, The Queen's University of Belfast, Belfast BT7 INN, United Kingdom
}

(Received 25 November 2015; accepted 15 January 2016; published online 1 February 2016)

\begin{abstract}
We demonstrate here a strong coupling between localized surface plasmon modes in self-standing nanorods with excitons in a molecular J-aggregate layer through angular tuning. The enhanced exciton-plasmon coupling creates a Fano like line shape in the differential reflection spectra associated with the formation of hybrid states, leading to anti-crossing of the upper and lower polaritons with a Rabi frequency of $125 \mathrm{meV}$. The recreation of a Fano like line shape was found in photoluminescence demonstrating changes in the emission spectral profile under strong coupling. (C) 2016 AIP Publishing LLC. [http://dx.doi.org/10.1063/1.4941078]
\end{abstract}

Developments in top-down and bottom-up nanofabrication techniques have enabled the development of active plasmonic nanomaterials such as arrays of gold nanorods with size- and shape-tunable plasmonic resonances. ${ }^{1-4}$ The active plasmonic nanomaterials can be coupled with excitonic systems to give rise to hybrid plasmon-exciton modes (Plexcitons) when in the strong coupling regime..$^{5-8}$ When such systems are within the strong coupling limit it creates an environment that induces changes in the optical processes of an emitter or absorber excitonic system. This offers potential to enhance or control exciton processes in excitonic systems such as organic semiconductors, which offers opportunities for enhanced photonic device designs such as in light harvesting, optical sensing, or artificial light sources. ${ }^{1,9,10}$ An understanding of light matter interactions of plasmonexciton complexes is central in fully realizing the potential of such complexes.

A number of studies have demonstrated plasmonexciton complexes in the strong coupling limit between an organic exciton semiconductor and surface plasmons $(\mathrm{SPs})^{11,12}$ and localized surface plasmons geometries. ${ }^{13-17}$ Previous studies have demonstrated that colloidal Au nanoshell-J-aggregate particles exhibit a strong coupling between the localized plasmons of a nanoshell and the excitons of molecular J-aggregates adsorbed on its surface. ${ }^{13}$ The interaction of an organic exciton in a J-aggregate and surface plasmon polariton modes of nanostructured hole arrays or of nanosize metallic disks with different array periods was reported to exhibit a strong coupling. ${ }^{14-16}$ Tuning of plasmo$\mathrm{n}$-exciton coupling strength has been reported for strongly coupled exciton-plasmon states in Au nanodisk arrays coated with J-aggregate molecules achieved by changing the incident angle of the incoming light, rather than changing the geometry of the plasmonic nanomaterial. Using such an angle resolved approach, the plasmon-exciton coupling of variable strengths was achieved. ${ }^{17}$

A strong coupling between plasmons on oriented gold nanorods arrays and J-aggregate molecular exciton has been demonstrated using a series of arrays with different

\footnotetext{
${ }^{\text {a) }}$ Author to whom correspondence should be addressed. Electronic mail: james.rice@ucd.ie. Telephone: 0035317172229
}

architectures to control the spatial and spectral overlap between the plasmonic structure and exciton molecular aggregates. ${ }^{18}$ Here, we demonstrate tuning/detuning of the strong coupling in the self-standing nanorod arrays achieved through angular tuning. We report a Fano line shape in the differential reflection spectra associated with the formation of hybrid states, leading to anti-crossing of the upper and lower cavity-polaritons with a Rabi frequency of $125 \mathrm{meV}$. The recreation of a Fano like line shape was also found in photoluminescence (PL), demonstrating changes in the emission spectral profile under strong coupling.

A schematic illustration of the sample, i.e., a quasiordered free-standing Au nanorod arrays, is shown in Figure 1(a). SEM studies (using a Jeol 6500F field emission SEM) reported that the $\mathrm{Au}$ nanorod substrate possesses a $70 \pm 11 \mathrm{~nm}$ array period (center to center distance), a rod diameter of $35 \mathrm{~nm} \pm 7$, and a rod height of $200 \pm 25 \mathrm{~nm}$. The nanorods are encased in an anodic aluminum oxide (AAO) template, which was removed prior to sample preparation via an etching solution in $30 \mathrm{mM} \mathrm{NaOH}$ as previously reported. ${ }^{3,4,18}$ J-aggregate samples were prepared using meso-tetra ( $n$-methyl-4-pyridyl) porphyrine tetra chloride (Frontier scientific) at $\mathrm{pH}=1$, inducing the formation of J-aggregate by drop deposition as per the procedures reported. ${ }^{19-21}$ Reflection spectra from the nanorod array were measured in TE and TM polarizations and showed two broad bands associated with transverse and longitudinal modes from the nanorod array, which are in line with literature reports (as shown in Figure 1(b)). ${ }^{3,4,18}$ The reflection spectra were recorded for the J-aggregate/nanorod sample as a function of $\left(\Delta R / R=\left(R_{\text {sample }}\right) / R_{\text {background }}\right)$ using a gold mirror background reference. An optical extinction spectrum (e.g., shown as $-\Delta R / R$ ) of a gold nanorod sample recorded is shown in Figure 1(b), showing the presence of transverse modes and longitudinal modes of the nanorod. Optical extinction spectroscopy of the porphyrin in the monomeric form was recorded along with the porphyrin in the Jaggregate form, as shown in Figure 1(b). The J-aggregate spectrum shows B (Soret or $\mathrm{S}_{2}$ exciton state) bands at 425 and $495 \mathrm{~nm} .{ }^{19}$ The spectrum also shows a Q-band $\left(\mathrm{S}_{1}\right.$ exciton state) region with a strong peak at $675 \mathrm{~nm}$. The redistribution of B and Q intensities following aggregation been attributed 

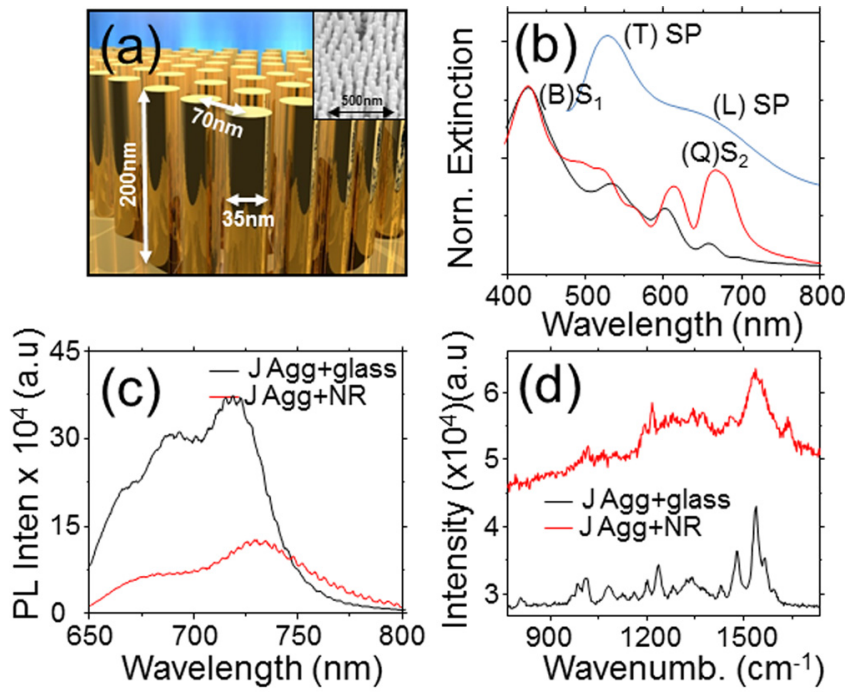

(e)

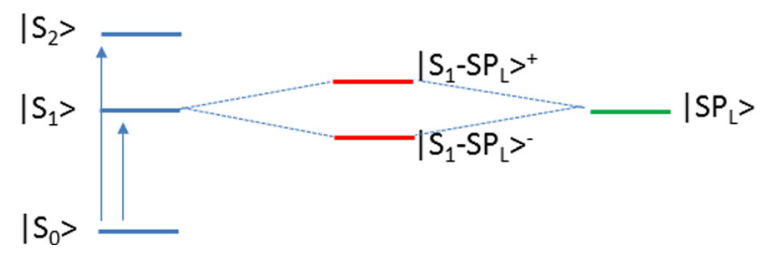

FIG. 1. (a) Schematic diagram of the nanorod array. (b) Extinction spectra of a J-aggregate thin film on a glass (red) shown along with a thin film of the initial monomer porphyrin (black) recorded using optical absorption spectroscopy, shown also is a spectrum for the nanorod sample (blue) recorded using an optical reflection spectroscopy, shown as the inverse of $\Delta R / R$. (c) Fluorescence spectroscopy of J-aggregate on a gold nanorod substrate $(\mathrm{J}-\mathrm{Agg}+\mathrm{NR})$ and on glass (J-Agg + glass) samples recorded at $470 \mathrm{~nm}$ in resonance with the J-aggregate B band. (d) Raman spectroscopy of J-aggregate on a gold nanorod substrate $(\mathrm{J}-\mathrm{Agg}+\mathrm{NR})$ and on glass (Por + glass) recorded at $532 \mathrm{~nm}$. (e) Schematic diagram of the electronic states for a strong coupling scheme showing the plexciton states.

to the intensity transfer from the B- to the Q-band region, which is mediated by an excitonic coupling between the $\mathrm{B}$ and Q transition dipoles.

Raman and fluorescence measurements were performed at ambient temperature using a custom-built, open-bench Raman system in epi-fluorescence backscattering configuration with 532, 473, and $633 \mathrm{~nm}$ excitation ${ }^{22-26}$ with the laser focused to a spot size of ca. $10 \mu \mathrm{m}$. The emission from the J-aggregate/nanorod complex is shown in Figure 1(c). The PL intensity for the J-aggregate/nanorod complex is reduced (quenched) compared to the J-aggregate on the dielectric glass. The fluorescence lifetime measurements were recorded, showing a reduction in the radiative lifetime (from 2.3 to $1.6 \mathrm{~ns}$ for the J-aggregate/nanorod sample compared to J-aggregate/glass). A change in the PL intensity and the spontaneous emission rate can be potentially attributed to the Purcell effect due to the local SP field, suggesting a weak coupling and/or to charge transfer of the exciton to the metal, resulting in enhanced non-radiative pathways for the J-aggregate samples. ${ }^{8}$ However, similar observations can also be made in the strong coupling limit. Raman spectroscopy of the porphyrin and J-aggregate samples on a gold nanorod substrate or glass is shown in Figure 1(d). Raman spectroscopy was performed at $532 \mathrm{~nm}$ in resonance with the Q band of both the J-aggregate samples and monomeric porphyrin resulting in a
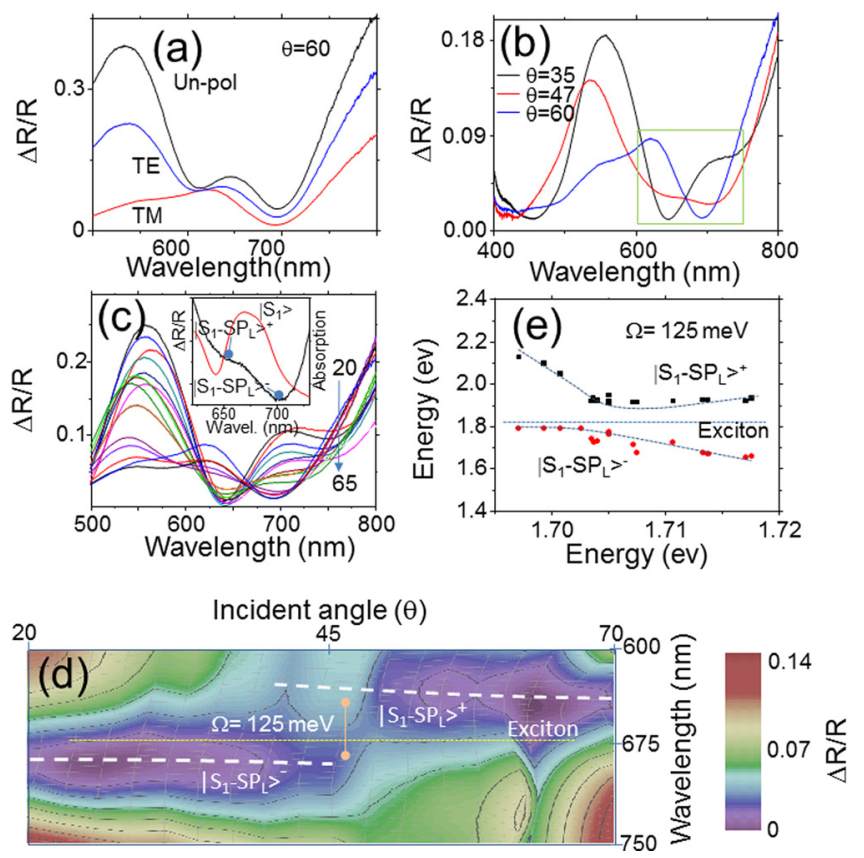

FIG. 2. Differential polarized reflection spectra for J-aggregate on a gold nanorod substrate. (a) Differential polarization spectra recorded with TE, $\mathrm{TM}$ and un-polarized light at an incidence angle of $60^{\circ}$. (b) Differential polarization spectra recorded with TM polarized light recorded at three different angles of incidence corresponding to the region of the nanorods SP modes and also of the exciton absorption of the J-aggregate. (c) Series of differential polarization spectra recorded with TM polarized light recorded at an incident angle between $20^{\circ}$ and $70^{\circ}$ recorded with $5^{\circ}$ intervals. Inset shows a single reflection spectrum from the J-aggregate-nanorod complex (recorded at $47^{\circ}$ ) along with the optical absorption spectrum of the J-aggregate. (d) Contour plot of the TM polarized reflection spectra at different incident angles. The spectra are shown between 600 and $750 \mathrm{~nm}$ corresponding to the nanorods longitudinal SP mode and also of the $\mathrm{S}_{1}$ absorption of the J-aggregate. (e) Dispersion curve of plexciton hybrid bands as a function of bare nanorod longitude SP position.

resonance enhancement in addition to the plasmonic enhancement. The Raman spectrum recorded on the nanorod substrate enhances the Raman signal strength. The origin of the increased signal intensity is assigned to plasmonic resonance Raman enhancement (also known as the surface enhanced resonance Raman spectroscopy or SERRS).

The reflection measurements in un-polarized and TE and TM polarized light for the J-aggregate/nanorod system were performed. Figure 2(a) shows a series of three spectra taken at a single incident angle $(\theta=$ degree) corresponding to three different polarizations. In line with previous studies, literature polarized reflection spectra showed that the TE polarized SP modes are at a lower energy (red-shifted) compared to the TM polarized SP modes, which are located at a higher energy due to a stronger restoring force of the charge oscillation. ${ }^{27}$ Figure 2(b) shows the differential reflectance $(\Delta \mathrm{R} / \mathrm{R})$ from the J-aggregate nanorod sample for the TM polarized light at three different incident angles. The three differential reflectance spectra show broad dips centered around 475 and $675 \mathrm{~nm}$, with the specific features at these regions dependent upon the angle of incidence. The dips in the reflection spectra are related to backscattering from SP associated with the transversal and longitudinal modes of the nanorod array. Concentrating on the wavelength range of $600-750 \mathrm{~nm}$ corresponds to a spectral region where the J-aggregate $S_{1}$ exciton state absorbs and also where the 
nanorods' longitudinal SP mode is present. To assess how the reflection spectra depends upon an angle, a more detailed series of measurements were performed with a consecutive series of reflection spectra recorded at every $2.5^{\circ}$ interval for incident angles from $20^{\circ}$ to $80^{\circ}$. Figures 2 (c) and 2(d) show the TM differential reflection spectra. At $\theta=35^{\circ}$, the spectra show a strong dip at ca. $645 \mathrm{~nm}$, and as the angle increases, this dip decreases in intensity, and a dip at ca. $705 \mathrm{~nm}$ emerges and grows in intensity as the angle increases. This is clearly seen in the contour plot (Figure 2(d)), which shows that there is a clear dip coinciding with the exciton energy replicating a Fano resonance line-shape, which indicates the presence of strong coupling. As schematically outlined in Figure 1(e) (and based on Salomon et al. ${ }^{16}$ ) in the strong coupling regime, plexcitons are formed by the interaction of the $\left|\mathrm{S}_{1}\right\rangle$ state of the exciton molecular J-aggregate with the longitudinal $\left(\left|\mathrm{SP}_{\mathrm{L}}\right\rangle\right)$ surface plasmon modes of the gold nanorod array. The hybrid plexcitons possess two branches (e.g., $\left|\mathrm{S}_{1}-\mathrm{SP}_{\mathrm{L}}\right\rangle^{+}$and $\left|\mathrm{S}_{1}-\mathrm{SP}_{\mathrm{L}}\right\rangle^{-}$). The reflection spectrum shows two dips marked $\left|\mathrm{S}_{1}-\mathrm{SP}_{\mathrm{L}}\right\rangle^{+}$and $\left|\mathrm{S}_{1}-\mathrm{SP}_{\mathrm{L}}\right\rangle^{-}$at $645 \mathrm{~nm}$ and $700 \mathrm{~nm}$, respectively (which corresponds to Rabi splitting $(\Omega)$ energy of $150 \mathrm{meV}$ ). Figures $2(\mathrm{c})$ and 2(d) show the $\mathrm{S}_{1} \mathrm{Q}$ band from the J-aggregates absorption spectrum, which has a peak at $675 \mathrm{~nm}$, which is approximately midway between the $\left|\mathrm{S}_{1}-\mathrm{SP}_{\mathrm{L}}\right\rangle^{+}$and $\left|\mathrm{S}_{1}-\mathrm{SP}_{\mathrm{L}}\right\rangle^{-}$hybrid states. To confirm the presence of strong coupling, a dispersion plot was made. A dispersion plot (Figure 2(d)) as a function of the single longitudinal SP mode of pristine nanorod with the plexciton hybrid modes of the J-aggregate/nanorod complex with an incident angle is shown in Figure 2(e). The dispersion plot shows a characteristic anti-crossing of the two hybrid peaks $\left(\left|\mathrm{S}_{1}-\mathrm{SP}_{\mathrm{L}}\right\rangle^{+}\right.$and $\left.\left|\mathrm{S}_{1}-\mathrm{SP}_{\mathrm{L}}\right\rangle^{-}\right)$. This is associated with Rabi splitting of the SP peak, where the molecular resonance of the Jaggregate and the longitudinal mode of the nanorod array overlaps the characteristic of the strong coupling.

The PL from the J-aggregate-nanorod complex was generated by tuning the excitation wavelength (at $632 \mathrm{~nm}$ ) towards being in resonant with the $\mathrm{Q}$ band of the J-aggregate centered at $675 \mathrm{~nm}$ arising from ${ }^{1,3} \mathrm{E}_{1} \leftarrow{ }^{1,3} \mathrm{E}_{3}$ with interporphyrin charge resonance transitions. Figures 3(a) and 3(c) show the fluorescence spectra recorded as a function of incident angle. The main peak in the florescence spectra shifts progressively to the blue, e.g., ca. $730(\theta=0)$ to $725(\theta=20)$ to $682(\theta=40)$ to $674(\theta=60) \mathrm{nm}$. This shift in position can be more clearly seen by subtraction of the J-aggregate emis$\operatorname{sion}(\theta=0)$ on glass from that of J-aggregate/nanorod at each angle (shown in Figure 3(b)). Following subtraction, the spectra show a dip at $715 \mathrm{~nm}$, and this dip corresponds to the position of the maximum band position from the reference $\mathrm{J}$-aggregate fluorescence spectrum recorded on a dielectric (glass) medium. Similar spectral profiles have been reported for PL from systems in the strong-coupling limit. ${ }^{12,27}$ Spectrally modified plasmon enhanced fluorescence or plasmon fluorescence quenching has been reported for molecular systems coupled with plasmonic structures. ${ }^{28}$ Such studies reported that the modification in the fluorescence profile is associated with the spectral dependence of the radiative and non-radiative decay rates of the molecular exciton complex closely follows the plasmon scattering spectrum. Figure 3(b) shows that the PL spectral profile moves to the blue with

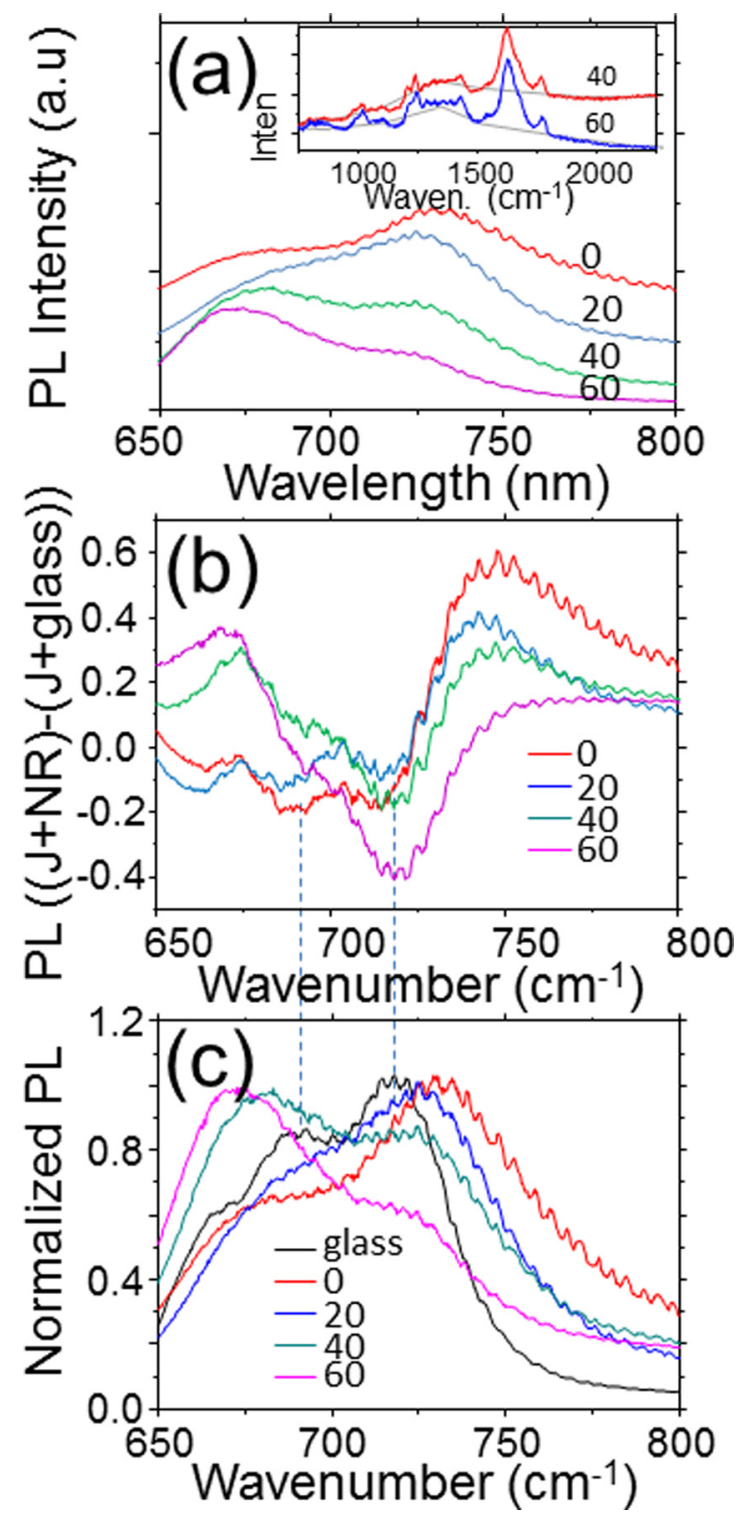

FIG. 3. PL spectroscopy of the J-aggregate on a gold nanorod substrate $\lambda_{\mathrm{ex}}=632 \mathrm{~nm}$. (a) PL spectra recorded at four different excitation angles. The inset shows SERS of the J-aggregate/nanorod complex for angles of excitation at $40^{\circ}$ and $60^{\circ} . \lambda_{\mathrm{ex}}=532 \mathrm{~nm}$. These angles are above and below the critical angle of ca. $47^{\circ}$ where the hybrid mode splits. (b) Subtraction of J-aggregate on a gold nanorod substrate spectra recorded at each of the four excitation angles shown in (a) from J-aggregate on a dielectric glass substrate. (c) Normalized overlaid PL spectra recorded from J-aggregate on a gold nanorod substrate recorded at each of the four excitation angles and J-aggregate on a dielectric glass substrate.

increasing incident excitation angle, which follows the observed shift in the reflection measurements (Figure 2). This indicates that the PL arises from the polaritonic emission, resulting from the plexciton SP/exciton mixed states.

Recently, it has been demonstrated that SP modes play a significant role in the observed Raman background in surface enhanced Raman spectroscopy (SERS). ${ }^{29}$ The authors reporting that the background in SERS comes from contributions centred strongest at the plasmon resonance regions in systems with localised SP modes such as in Au nanorod arrays. The SP modes provide the strongest out-coupling of the radiation from the electrons relaxing back down from the virtual inelastic light scattering state. The spectral resonance of the plasmons thus dictates which energy range of 
electrons contributes to the Raman (Stokes) background. SERRS spectroscopy was performed to assess the impact of angle. Figure 3(a)-inset shows SERS spectra recorded at $532 \mathrm{~nm}$. A base line formation routine (Origin Labs) to highlight the background on which the spectra are located was used to assess the contribution of the background with regard to the spectral profile. The resulting spectra show that there is a change in the background profile. Looking at the background, it can be seen that the background shifts to the blue with increasing angle similar to that seen for the PL spectra.

In summary, we demonstrate here strong coupling between localised surface plasmon modes in self-standing nanorods with Frankel excitons in a porphyrin molecular Jaggregate layer through angle tuning. The enhanced exciton-plasmon coupling creating a Fano line shape in the differential reflection spectra associated with the formation of plexciton hybrid states, leading to anti-crossing of the upper and lower cavity-polaritons with a Rabi frequency of $125 \mathrm{meV}$. The recreation of a Fano like line shape was found in photoluminescence demonstrating changes in the emission spectral profile under strong coupling. This study demonstrates that J-aggregate excitons under strong coupling with plasmon modes can controllably alter the emission and absorption spectral profile through control of excitation angle.

The UCD Nanophotonics and Nanoscopy Research Group was supported by Science Foundation Ireland grant 12/IP/1556. The Centre for Nanostructured Media acknowledges the Engineering and Physical Sciences Research Council (EPSRC - UK) for financial support (Grant Nos. EP/H000917 and EP/I014004).

\footnotetext{
${ }^{1}$ Active Plasmonics and Tuneable Plasmonic Metamaterials, edited by Anatoly V. Zayats and Stefan Maier (Wiley, 2013).

${ }^{2}$ A. V. Kabashin, P. Evans, S. Pastkovsky, W. Hendren, G. A. Wurtz, R. Atkinson, R. Pollard, V. A. Podolskiy, and A. V. Zayats, Nat. Mater. 8, 867-871 (2009).

${ }^{3}$ S. Damm, S. Fedele, A. Murphy, K. Holsgrove, M. Arredondo, R. Pollard, and J. H. Rice, Appl. Phys. Lett. 106, 183109 (2015).
}

${ }^{4}$ S. Damm, F. Lordan, A. Murphy, M. McMillen, R. Pollard, and J. H. Rice, Plasmonics 9, 1371-1376 (2014).

${ }^{5}$ W. L. Barnes, A. Dereux, and T. W. Ebbesen, Nature 424, 824 (2003).

${ }^{6}$ A. F. Koenderink, A. Alù, and A. Polman, Science 348, 516 (2015).

${ }^{7}$ L. Novotny, Am. J. Phys. 78, 1199 (2010).

${ }^{8}$ E. M. Purcell, Phys. Rev. 69, 37 (1946).

${ }^{9}$ C. Gonzalez-Ballestero, J. Feist, E. Moreno, and F. J. Garcia-Vidal, Phys. Rev. B 92, 121402(R) (2015).

${ }^{10}$ J. Zhao, L. J. Sherry, G. C. Schatz, and R. P. Van Duyne, IEEE J. Quantum Electron. 14, 1418 (2008).

${ }^{11}$ J. Bellessa, C. Bonnand, J. C. Plenet, and J. Mugnier, Phys. Rev. Lett. 93, 036404 (2004).

${ }^{12}$ C. Symonds, J. Bellessa, J. C. Plenet, A. Bréhier, R. Parashkov, J. S. Lauret, and E. Deleporte, Appl. Phys. Lett. 90, 091107 (2007).

${ }^{13}$ N. T. Fofang, T. H. Park, O. Neumann, N. A. Mirin, P. Nordlander, and N. J. Halas, Nano Lett. 8, 3481-3487 (2008).

${ }^{14}$ J. Dintinger, S. Klein, F. Bustos, W. L. Barnes, and T. W. Ebbesen, Phys. Rev. B 71, 035424 (2005).

${ }^{15}$ J. Bellessa, C. Symonds, K. Vynck, A. Lemaitre, A. Brioude, L. Beaur, J. C. Plenet, P. Viste, D. Felbacq, and E. Cambril, Phys. Rev. B 80, 033303 (2009).

${ }^{16}$ A. Salomon, S. Wang, J. A. Hutchison, C. Genet, and T. W. Ebbesen, ChemPhysChem 14, 1882-1886 (2013).

${ }^{17}$ Y. B. Zheng, B. K. Juluri, L. Lin Jensen, D. Ahmed, M. Lu, L. Jensen, and T. J. Huang, Adv. Mater. 22, 3603-3607 (2010).

${ }^{18}$ G. A. Wurtz, P. R. Evans, W. Hendren, R. Atkinson, W. Dickson, R. J. Pollard, and C. Bower, Nano Lett. 7, 1297-1303 (2007).

${ }^{19}$ M. H. Reddy, R. Al-Shammaria, N. Al-Attar, S. Lopez, T. Keyes, and J. H. Rice, Mater. Res. Express 1, 045038 (2014).

${ }^{20}$ H. V. Reddy, R. M. Al-Shammaria, N. Al-Attara, E. Kennedy, L. Rogers, S. Lopezc, T. E. Keyes, M. O. Senge, and J. H. Rice, Phys. Chem. Chem. Phys. 16, 4386-4393 (2014).

${ }^{21}$ M. H. V. Reddy, R. M. Al-Shammari, N. Al-Attar, L. Rogers, S. Lopez, R. J. Forster, and J. H. Rice, Chem. Phys. Mater. 143, 963-968 (2014).

${ }^{22}$ F. Lordan, J. H. Rice, B. Jose, R. J. Forster, and T. E. Keyes, J. Phys. Chem. C 116, 1784-1788 (2012).

${ }^{23}$ S. Damm, N. C. Carville, B. J. Rodriguez, M. Manzo, K. Gallo, and J. H. Rice, J. Phys. Chem. C 116, 26543-26550 (2012).

${ }^{24}$ N. Al-Attar, I. Kopf, E. Kennedy, K. Flavin, S. Giordani, and J. H. Rice, Chem. Phys. Lett. 535, 146-151 (2012).

${ }^{25}$ S. Damm, N. C. Carville, M. Manzo, K. Gallo, S. G. Lopez, T. E. Keyes, and J. H. Rice, Appl. Phys. Lett. 103, 083105 (2013).

${ }^{26}$ S. Najmaei, A. Mlayah, A. Arbouet, C. Girard, J. Léotin, and J. Lou, ACS Nano 8, 12682-12689 (2014).

${ }^{27}$ B. Lee, J. Park, G. H. Han, H. S. Ee, C. H. Naylor, W. Liu, and R. Agarwal, Nano Lett. 15, 3646-3653 (2015).

${ }^{28}$ E. C. Le Ru, P. G. Etchegoin, J. Grand, N. Felidj, J. Aubard, and G. Levi, J. Phys. Chem. C 111, 16076-16079 (2007).

${ }^{29}$ J. T. Hugall and J. J. Baumberg, Nano Lett. 15, 2600-2604 (2015). 